\title{
VSOP, A SPACE VLBI PROGRAMME
}

\author{
H. HIRABAYASHI \\ The Institute of Space and Astronautical Science \\ 3-1-1 Yoshinodai, Sagamihara, Kanagawa 299, Japan
}

\begin{abstract}
VSOP, VLBI Space Observatory Programme, is an approved space VLBI programme of ISAS for the study of very compact radio sources with the synthesized aperture of $30,000 \mathrm{~km}$ diameter, by connecting an orbiting radio observatory with ground radiotelescopes. The VSOP satellite carrying $10 \mathrm{~m}$ antenna with $1.6,5$, and $22 \mathrm{GHz}$ band receivers will be launched in early 1995 by M-V rocket of ISAS into an eccentric orbit with 20,000 $\mathrm{km}$ in apogee height. The tracking network will be formed for the satellite orbit determination, phase transfer and IF down-link. VSOP aims imaging capability with best resolution of 0.0001 arc second in $22 \mathrm{GHz}$ band. Imaging of active galactic nuclei, star forming regions and stellar objects, and radioastrometry are main scientific targets.
\end{abstract}

\section{Introduction}

VLBI observations provide very high angular resolution of the order of 1 milliarcsecond. But serious limits of this outstanding technique is still in angular resolution and picture quality, because the element antennas are fixed on the Earth. Obvious solution to this is having at least one element antenna in space. That this is technically feasible and scientifically valuable has been successfully demonstrated by the TDRSS Orbiting VLBI experiments in 1986 through 1988 (Levy et al., 1986), (Linfield et al., 1990) and (Linfield et al., 1989). In this experiment, the local oscillator onboard the satellite was phase-locked to the ground based hydrogen maser frequency standard and IF signal was down linked to the ground for recording.

The Institute of Space and Astronautical Science (ISAS) has a funded space VLBI programme called VSOP, A VLBI Space Observatory Programme. First, the programme assumed M-3SII rocket for the launching vehicle and the satellite were more modest (Hirabayashi, 1987), but the approval for the new rocket in ISAS resulted in a satellite with reasonable size and sensitivity. The VSOP satellite launch will be in early 1995 and participation from institutes worldwide is assumed. The launch of Soviet Radioastron (Kardashev and Slysh,1987) for space VLBI is reported to occur mid 1990.

The VSOP international symposium was held in ISAS in December 1989, and the proceedings of the symposium is in press with the title "Frontiers of VLBI". So the readers are suggested to consult the book for more detail.

\section{General Concepts of VSOP and Science Targets}

VSOP will use an orbiting radio observatory with a $10 \mathrm{~m}$ antenna in an eccentric orbit to be coherently connected to the radiotelescopes widely spread on the earth. The phase reference signal for local oscillators onboard will be uplinked from the hydrogen masers on the ground and return signal will be compared for two way

Y. Kondo (ed.), Observatories in Earth Orbit and Beyond, 263-269.

(C) 1990 Kluwer Academic Publishers. Printed in The Netherlands. 


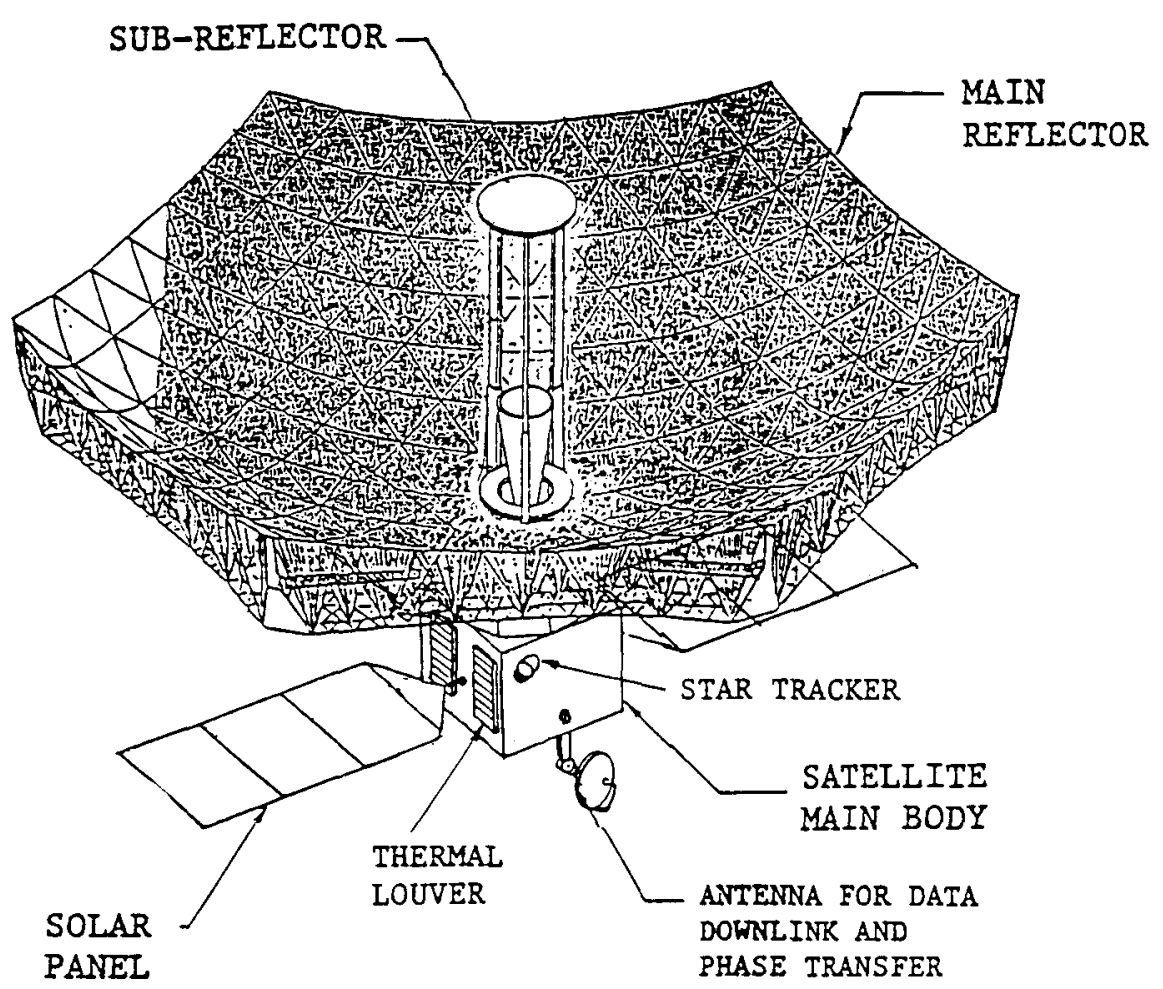

Fig. 1. Present sketch of the Muses-B spacecraft for VSOP

phase monitor and for Doppler tracking. While the IF signal with $64 \mathrm{MHz}$ maximum bandwidth will be downlinked digitally to be recorded to magnetic tapes for later correlation and image processing. The basic scheme of this aperture synthesis is common with the ground based VLBI. The difference is only in the presence of telemetry link for the satellite.

The maximum aperture of $30,000 \mathrm{~km}$ will be synthesized to give the best angular resolution of 0.1 milli-arcsecond at $22 \mathrm{GHz}$. Table 1 is for spatial resolution and scientific rational.

The most important targets of VSOP are Active Galactic Nuclei (AGN). Already by the TDRSS experiment, the sources exceeding the Compton limit of $10 \mathrm{E} 12$ $\mathrm{K}$ were detected proving the relativistic beaming hypothesis. The projected baseline length of TDRSS experiment and VSOP are the same, but due to the very limited coverage of the UV-coverage, mapping was left undone. The resolution of VSOP is comparable to ground mm-VLBI. VSOP and mm-VLBI will make the accreting, beaming, and radiation mechanisms clear.

Compact stellar objects are accessible by VSOP. Maser sources either in the star forming stage or in late type stage can be imaged and monitored by $\mathrm{OH}$ and $\mathrm{H} 2 \mathrm{O}$ maser lines. By proper motion measurements of maser spots, we can determine the distance to the region better than by ground VLBI. Pulsars are another group of 


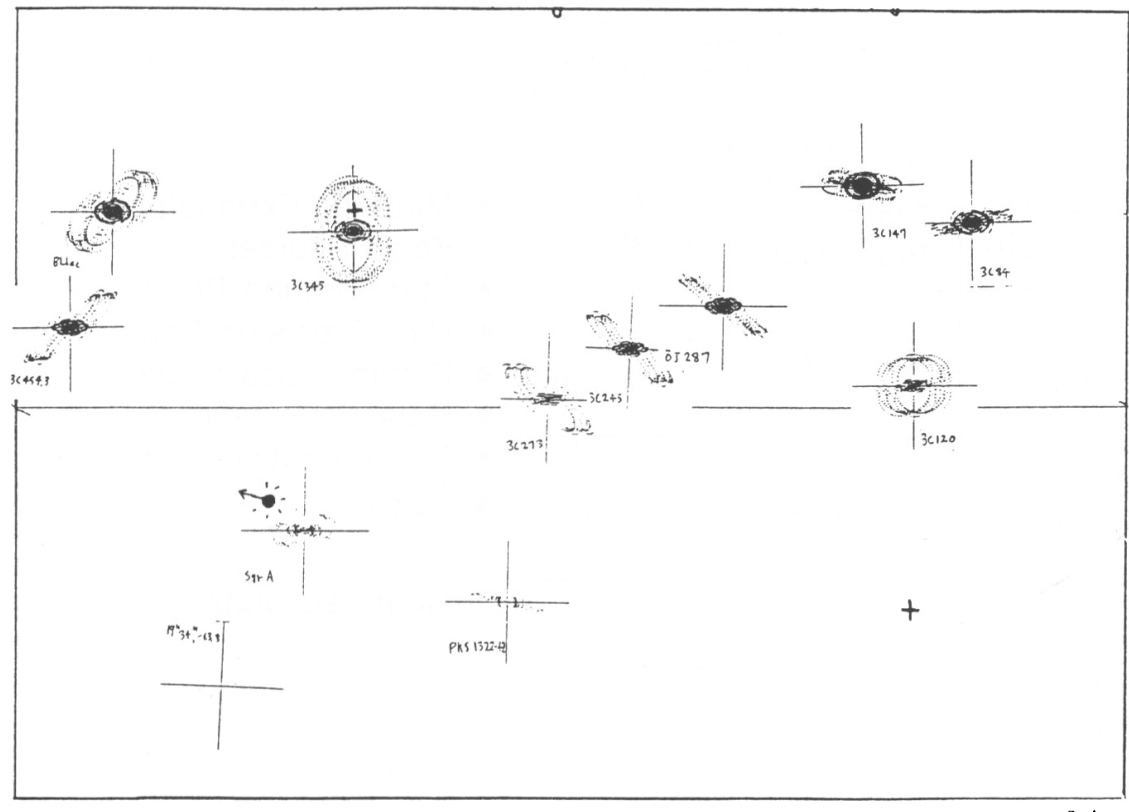

Fig. 2. U-V coverages of typical AGNs in a whole sky map at a fixed epoch
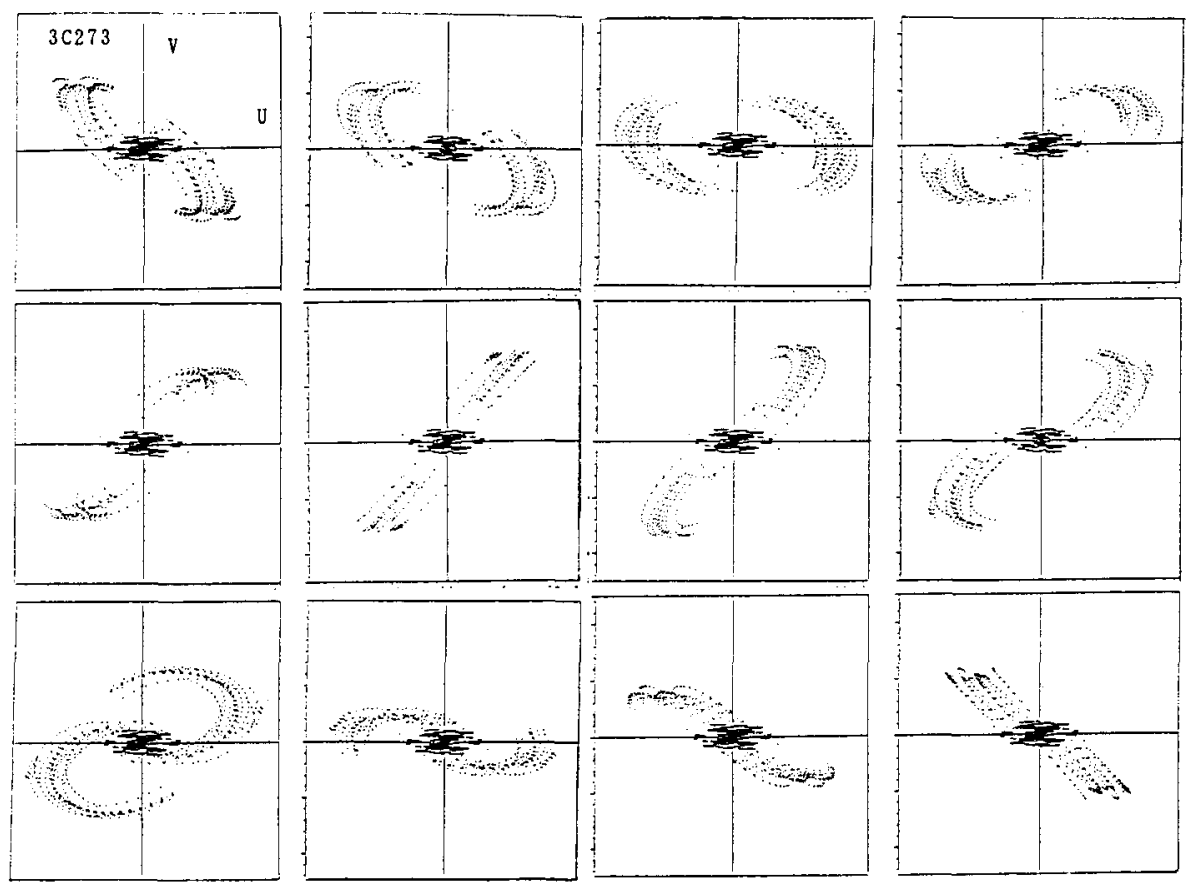

Fig. 3. Time variation of UV-coverage of $3 \mathrm{C} 273$ every 2 months. 
TABLE I

Observing frequency, maximum angular resolution, and scientific rationale.

\begin{tabular}{|c|c|c|c|}
\hline $\begin{array}{c}\text { Frequency } \\
\text { (Wavelength) }\end{array}$ & $\begin{array}{l}\text { Minimum } \\
\text { Synthesized } \\
\text { Beamwidth }\end{array}$ & $\begin{array}{l}\text { Scientific } \\
\text { Objective }\end{array}$ & - Mapping Extragalactic \\
\hline $\begin{array}{l}22 \mathrm{GHz} \\
(1.3 \mathrm{~cm})\end{array}$ & $0.13 \mathrm{mas}^{*}$ & $\begin{array}{l}\mathrm{H}_{2} \mathrm{O} \text { maser } \\
\text { continuum }\end{array}$ & $\begin{array}{l}\text { - High Precision Radioastrometry } \\
\text { - Distance Scale determination } \\
\text { - Galactic Rotation }\end{array}$ \\
\hline $\begin{array}{l}5 \mathrm{GHz} \\
(6 \mathrm{~cm})\end{array}$ & 0.6 mas & contnuum & $\begin{array}{l}\text { - Low rrequency Variables } \\
\text { - Pulsars }\end{array}$ \\
\hline $\begin{array}{l}1.7 \mathrm{GHz} \\
(18 \mathrm{~cm})\end{array}$ & $1.8 \mathrm{mas}$ & $\begin{array}{l}\text { OH maser } \\
\text { continuum }\end{array}$ & milli arc seconds \\
\hline
\end{tabular}

stellar sources to be studied.

Radioastrometry can be done by the extension of the baseline lenght but not with the better knowledge of the satellite orbit. Socalled delta-VLBI can be done in some cases.

The initial astronomical requirements are discussed by Hirabayashi (1991a).

\section{Muses-B Satellite for VSOP}

The fabrication and the launch of the VSOP satellite, currently named as Muses$\mathrm{B}$, started in 1989. New M-V rocket of ISAS will be used for launching. The M-V rocket will consist of three stage solid propellant boosters and will be able to carry about 2 tons payload to the low earth orbit (LEO). The development of the $\mathrm{M}-\mathrm{V}$ rocket started from 1990.

Total payload mass will be about $800 \mathrm{~kg}$ and the total electric power in the beginning of life (BOL) will be about $550 \mathrm{~W}$. The power will be generated by two solar paddles extended in opposite directions from the satellite main body. Figure 1 show the spacecraft of the present design.

The $10 \mathrm{~m}$ radio astronomy antenna fixed to the satellite main body will be deployed after launch. The antenna structure is a so-called tension truss design with six extension booms as main back structure, and the reflecting surface is made of wire mesh. The antenna is center fed Cassegrain type, and has 1.6, 5 and $22 \mathrm{GHz}$ feeds. The sub-reflector also will be extended to the nominal position after deployment. 1.6 and $5 \mathrm{GHz}$ frontends will be passively cooled, and $22 \mathrm{GHz}$ frontend will be actively cooled down to about $80 \mathrm{~K}$ by Stirling cycle refrigerator.

There will be two independent LO and IF channels for radioastronomy signal. 
The local oscillators for the mixers will be generated from synthesizers phase-locked to the reference signal sent from the ground telemetry network. The two IF channels are followed by video-converters and A/D converters independently. Again local synthesizers are phase locked to the reference signal from the ground. A/D converters are 1 or 2 bit mode and with $32 \mathrm{MHz}$ or $16 \mathrm{MHz}$ bandwidth. A formatter unit accepts the digital data from the two A/D converters to combine a bit stream with maximum rate of $128 \mathrm{Mbps}$. This will be formatted with synchronizing, timing and auxiliary bits. The bit streams will be QPSK modulated and down-linked through a power amplifier and a $40 \mathrm{~cm}$ high gain antenna in $\mathrm{Ku}$-band. As the wideband down link is digitally coded, the phase fluctuation from atmosphere in downlink path is negligible.

The reference signal from hydrogen masers on the ground will be up-linked in Ku-band for the frequency standards on-board. The Ku-band on-board antenna receives the signal, and the signal will be transmited back to the ground to monitor the phase variation and for precise Doppler tracking. The $40 \mathrm{~cm}$ antenna will be equiped on a gimbal at the bottom of the satellite bottom.

The satellite will be 3 -axis stabilized, and the pointing accuracy of the radioastronomy antenna is 0.01 degree, and this will be managed by the Reaction Control System (RCS) with star sensors, inertial-gyro and torque motor. The satellite will have thrusters for perigee-up, and angular momentum compensation, etc.

Due to the oblateness of the earth, the argument of perigee and the ascending node of the orbit change. These are about one revolution per 2 years. This means that good direction of the sky for observing changes with time. Observing schedule will be made with this effect in mind.

More details of VSOP satellite and on-board radioastronomy instruments are treated by Hirosawa (1991) and Hirabayashi (1991b).

\section{Ground Supports for VSOP and Observing}

For VSOP, two kinds of ground networks are necessary; one for the satellite; commanding and housekeeping, orbit determination, and for phase transfer and wideband data link. The other is array of co-observing radio telescopes.

ISAS will provide a tracking station by using $20.9 \mathrm{~m}$ antenna at Kagoshima Space Center (KSC). This will be used for the commanding and housekeeping in S-band, and for telemetry in $\mathrm{Ku}$-band. $64 \mathrm{~m}$ antenna at Usuda (ISAS), $45 \mathrm{~m}$ radiotelescope at Nobeyama ( $\mathrm{NRO}$, Nobeyama Radio Observatory), and $34 \mathrm{~m}$ antenna at Kashima (CRL, Communication Research Laboratory) are the candidates for co-observing stations in Japan.

Deep Space Network(DSN) of NASA/JPL will support VSOP by building telemetry network with $10 \mathrm{~m}$ dedicated antennas at each DSN site. The link power budget for spacecraft is so designed that $10 \mathrm{~m}$ antenna can handle the satellite. The Green Bank telemetry station of NRAO also is planned and this, too, helps the VSOP telemetry coverage.

Arraying with VLBI network telescopes like VLBA (Very Long Baseline Interferometer Array, US), EVN (European VLBI Network, Europe), and AT (The Australia Telescope, Australia) and other non-network telescopes are assumed. VLBA 
is a longer baseline array and will be suitable for high fidelity maps for stronger sources. AT will be essential for southern radio sources with VSOP because VSOP will be the first all-sky mapping instrument.

The tms sensitivities for VSOP-ground interferometer with $64 \mathrm{MHz}$ bandwith, $100 \mathrm{~s}$ integration time are about $10 \mathrm{~m}$ Jy for $64-70 \mathrm{~m}$ class ground antenna and $25 \mathrm{~m}$ class ground antenna. Interference fringe several times larger than these level cauld be detected.

For correlating the observed tapes and for sophisticated aperture synthesis imaging, A 10-20 station correlator of FX architecture, with a super computer for imaging capability has been proposed and design study is in progress. We hope the correlator will be partially operational in 1993.

Formation of some kind of worldwide consortium for VSOP is needed in many repects, and discussion is in progress. Inter Agency Consultative Group (IACG) of Space Science Panel-1 meting has been dealing the operational management inviting the representatives from ground telescope arrays.

\section{Possible observing scenario}

The image quality depends on the UV-coverage, the optical transfer function, and it is a function of source direction and the epoch. The initial orbit and the ground facilities are primary important parameters. The orbit plane changes with $d W / d t=$ -177 degrees/year and $d w / d t=177$ degrees/year where $\mathrm{W}$ is right ascension of ascending node and $w$ is argument of perigee. There are two dominant constraint factors in the satellite which limit the UV-coverage. One is that the satellite cannot observe the direction of the Sun with 70 degrees cone angle, because solar paddles cannot be well illuminated due to the shadow of the $10 \mathrm{~m}$ antenna. The other is the tracking coverage of $\mathrm{Ku}$-band downlink antenna to point telemetry network.

For observing, above constraints must be carefully taken into account. Figure 2 shows an example of UV-coverages of typical AGNs on all sky map at an epoch. Kagoshima and 3 DSN stations are assumed for telemetry network and VLBA.

An example of time variation of UV-coverage is shown in Figure 3 for $3 \mathrm{C} 273$. It is seen that the UV-coverage significantly changes with time. So, if we monitor such source, care should be taken for the beam shape and sensitivity changes while interpretation. The coverage recurrs after about 2 years due to the orbit change, and with 2 year span we can compare the radio source structure change very precisely.

\section{Discussion}

The Soviet Radioastron satellite also will carry a $10 \mathrm{~m}$ antenna, and its orbit is $7,400 \mathrm{~km}$ in perigee and $77,000 \mathrm{~km}$ in apogee. This aims higher angular resolution but less imaging capability if compared to VSOP. The Radioastron is a detection instrument, while VSOP is a mapping instrument, and the two are astrophysically complimentary. If the operational time overlaps between VSOP and Radioastron, we can combine the visibility data, making better UV-coverage for better image of radio sources. 
For a limited number of strong sources, there is the possibility of getting fringes between the two satellites. Also, VSOP may be used to calibrate the visibility data of Radioastron-ground system using hte overlaping part of VSOP-Radioastron visibility data. In any case, both space VLBI programmes need ground resources common in nature, and the compatibility of scheme and instruments is an important issue.

Even though VSOP will start new sciences in space, it is still limited in sensitivity. With better sensitivity, we have better image, more sources, and more science. VSOP and Radioastron will answer some astrophysical questions, and discover new questions, and second generation missions have already have firm reason for existence.

\section{Conclusion}

The concept of VSOP and scientific targets are shown briefly. As a first geneation space-VLBI observatory, VSOP will make first step for the understanding of very compact and bright objects in the universe.

\section{References}

Levy, G.S. et al.: 1986, Science 234, 187

Linfield, R. et al.: 1990, Astrophys. Jour. 358, 350

Linfield, R. et al.: 1989, Astrophys. Jour. 336, 1105

Hirabayashi, H.: 1987, in M.R. Reid and J.M. Moran, ed(s)., Proceedings of IAU Symposium No.129, The Impact of VLBI on Astrophysics and Geophysics, Reidel, Dordrecht, 441

Kardashev, N.S. and Slysh, V.I.: 1987, in M.R. Reid and J.M. Moran, ed(s)., Proceedings of IAU Symposium No.129, The Impact of VLBI on Astrophysics and Geophysics, Reidel, Dordrecht

Hirabayashi, H.: 1991, Hirabayashi, H., Kobayashi, H. and Inoue, M., Frontiers of VLBI, Universal Academy Press, Tokyo

Hirosawa, H.,1991, ibid

Hirabayashi, H., 1991,ibid 